\title{
A Local Coupling Multi-Trace Domain Decomposition Method for Electromagnetic Scattering from Multilayered Dielectric Objects
}

\author{
Ran Zhao, Member, IEEE, Yongpin Chen, Senior Member, IEEE, \\ Xian-Ming Gu, Zhixiang Huang, Senior Member, IEEE, Hakan Bagci, Senior Member, IEEE, \\ and Jun Hu, Senior Member, IEEE
}

\begin{abstract}
In this paper, a local coupling multi-trace domain decomposition method (LCMT-DDM) based on surface integral equation (SIE) formulations is proposed to analyze electromagnetic scattering from multilayered dielectric objects. Different from the traditional SIE-DDM, where the interactions between sub-domains are accounted for using global radiation coupling, LCMT-DDM uses a local coupling scheme. The original multilayered object is decomposed into several independent domains, i.e. the exterior region (free space) and many homogeneous interior regions (dielectrics). The boundaries of sub-domains are all touching-faces, where only the Robin transmission conditions (RTCs) are enforced to ensure the field continuity. Hence, each sub-domain only couples with its neighboring regions, which makes the DDM system a highly sparse matrix especially when the number of sub-domains is large. In each sub-domain, the electric field integral equation (EFIE) and the magnetic field integral equation (MFIE) for dielectrics are used as the governing equations. By imposing RTCs, well-conditioned equations are formed in each sub-domain without invoking the combined field integral equation (CFIE), which usually causes accuracy issues in dielectric modeling. Since the sub-domain matrices are diagonally dominant, the flexible generalized minimal residual (FGMRES) technique is used to accelerate the iterative solution of the whole DDM system. Moreover, an effective preconditioner that can be recursively constructed is proposed.
\end{abstract}

Index Terms-Electromagnetic scattering, local coupling, domain decomposition, multi-trace, multilayered, dielectric.

\section{INTRODUCTION}

A CCURATE solution of electromagnetic (EM) scattering from multilayered dielectric objects is of vital importance

This work was supported in part by NSFC No. 61801002, No. 61722101, No. 61871079, No. 61801097, No. 11801463 and in part by NSF of Anhui Province No. 1808085QF183, No. KJ2018A0015. (Corresponding author: Yongpin Chen.)

Ran Zhao and Zhixiang Huang are with the Key Laboratory of Intelligent Computing and Signal Processing, Ministry of Education, Anhui University, Hefei Anhui 230039, China.

Yongpin Chen and Jun $\mathrm{Hu}$ are with School of Electronic Science and Engineering, University of Electronic Science and Technology of China (UESTC), Chengdu 611731, China.

Xian-Ming Gu is with School of Economic Mathematics, Southwestern University of Finance and Economics, Chengdu, Sichuan 611130, China.

Ran Zhao is also with the Division of Computer, Electrical, and Mathematical Science and Engineering (CEMSE), King Abdullah University of Science and Technology (KAUST), Thuwal, Saudi Arabia.

Hakan Bagci is with the with the Division of CEMSE, KAUST, Thuwal, Saudi Arabia. (e-mail: rzhao1988@foxmail.com, ypchen@uestc.edu.cn, guxm@swufe.edu.cn, zxhuang@ahu.edu.cn, hakan.bagci@kaust.edu.sa, hujun@uestc.edu.cn) in various applications, such as target identification, antenna design, and microwave circuit analysis, etc. The full-wave method of moments (MoM) is one of the most widely used methods due to its robustness, efficiency, and accuracy [1], [2], [3]. When the volume integral equation (VIE) is used, a large number of unknowns are inevitably generated because of volumetric discretization, especially for large-scale problems [4], [5], [6]. For homogeneous or piecewise homogeneous objects, surface integral equation (SIE), which requires only the surfaces to be discretized is usually a better choice [7], [8].

However, when SIE is used to model piecewise homogeneous or multilayered dielectric objects, careful treatment of complex junction basis functions is required to ensure the boundary conditions [9]. To overcome this difficulty, the contact-region modeling method (CRM) [10] and domain decomposition method (DDM) [11], [13], [14] have been developed. In CRM, the interface is divided into two contact surfaces to ensure the boundary condition between different regions. Although CRM can automatically guarantee the continuity of electric and magnetic fields at the interface, it always leads to an ill-conditioned matrix that can not be solved efficiently using an iterative solver. Similar to CRM, DDM also decomposes the original piecewise homogeneous dielectric objects into different sub-domains. Nevertheless, the boundary conditions in DDM are ensured by imposing the transmission conditions (TCs). Moreover, the resultant DDM matrix is usually well conditioned.

In the framework of DDM, the combined field integral equation (CFIE), such as the electric-magnetic field CFIE (JMCFIE) [15], [16] and generalized-CFIE (GCFIE) [12], are commonly used as the governing equations on each subdomain . Compared to the traditional Poggio-Miller-ChangHarrington-Wu-Tsai (PMCHWT) equation [2], the iterative convergence of CFIE is much faster. This plays an important role in DDM, since the well-conditioned sub-domain matrices can be used as an preconditioner for the entire system. However, this favorable iterative convergence is achieved at the expense of compromised accuracy inherited from CFIE [17]. Moreover, due to the global radiation coupling among different sub-domains, full matrices are generated in the conventional SIE-DDM system.

To address the accuracy issue of the CFIE based DDM, a novel local coupling multi-trace domain decomposition 
method (LCMT-DDM) is proposed in this work. This method first decomposed the original object into two sub-domains, the exterior region and the interior one. Then, the interior region is further decomposed, where each dielectric layer is defined as one sub-domain. By doing this, the boundaries of each subdomain are always the touching faces. Since each sub-domain is meshed independently, there are always a pair of meshes at the sub-domain interfaces, which is referred to as multitraces [19], [20]. It is noted that in traditional SIE-DDM and $\mathrm{CRM}$, the interactions between sub-domains are accounted for using global radiation coupling. On the other hand, the proposed LCMT-DDM, couples each sub-domain only to its neighbors using the Robin transmission conditions (RTCs). After discretization, this local coupling scheme leads to a sparse DDM matrix and reduces the memory consumption, especially for multilayered dielectric objects.

In each sub-domain, EFIE and MFIE are simultaneously used as the governing equations to ensure the accuracy and to eliminate the interior resonance problem. However, neither EFIE nor MFIE is well-conditioned [21]. Fortunately, when they are combined with RTCs, the resultant matrix can be made diagonally dominant and well-conditioned, which is very similar to the structure of CFIE. Hence, the sub-domain matrices are used as the preconditioner in the FGMRES solver, which is based on an inner-outer iteration scheme [22], [23]. It is noted that this preconditioner does not require extra memory, in contrast with the traditional sparse approximate inverse (SAI) preconditioner, or the incomplete lower-upper factorization preconditioner [24], [25]. Moreover, FGMRES is compatible with these conventional preconditioners since they can also be applied during the inner iteration. In this work, the multilevel fast multiple algorithm (MLFMA) is applied in FGMRES to accelerate the computation of matrix-vector products required by the FGMRES solver[26].

The rest of this paper is organized as follows. In Section II, the detailed derivation of the LCMT-DDM equations are provided. Then the discretization, matrix system formulation and preconditioning techniques are discussed. In Section III, numerical examples are presented to demonstrate the performance of LCMT-DDM. Conclusions are summarized and future research directions are provided in Section IV.

\section{FORMULATION}

\section{A. LCMT-DDM Formulation}

Consider the EM scattering problem as shown in Fig. 1, where a piecewise homogeneous structure $\Omega_{d}$ is illuminated by an incident plane wave $\left\{\mathbf{E}^{i n c}, \mathbf{H}^{i n c}\right\}$. The regions $\Omega_{d}$ is a combination of $\Omega_{d 1}$ and $\Omega_{d 2}$, satisfying $\Omega_{d}=\Omega_{d 1} \cup \Omega_{d 2}$, $\Omega_{d 1} \cap \Omega_{d 2}=\emptyset$, where $\Omega_{d 1}$ and $\Omega_{d 2}$ are two homogeneous regions with relative permittivity, permeability $\varepsilon_{r 1}, \mu_{r 1}$ and $\varepsilon_{r 2}, \mu_{r 2}$ respectively. The background is the free space with permittivity, permeability $\varepsilon_{0}, \mu_{0}$. The scattered fields are denoted as $\left\{\mathbf{E}^{s c a}, \mathbf{H}^{s c a}\right\}$, and the equivalent electric current $\mathbf{J}$ and the scaled magnetic current $\mathbf{M}$ satisfy $\mathbf{J}=\hat{\mathbf{n}} \times\left(\mathbf{H}^{i n c}+\right.$ $\left.\mathbf{H}^{s c a}\right)$ and $\mathbf{M}=\frac{1}{\eta_{0}}\left(\mathbf{E}^{i n c}+\mathbf{E}^{s c a}\right) \times \hat{\mathbf{n}}$, where $\eta_{0}=\sqrt{\frac{\mu_{0}}{\varepsilon_{0}}}$ is the intrinsic impedance of free space. As shown in Fig. 2, the original computational domain is decomposed into three

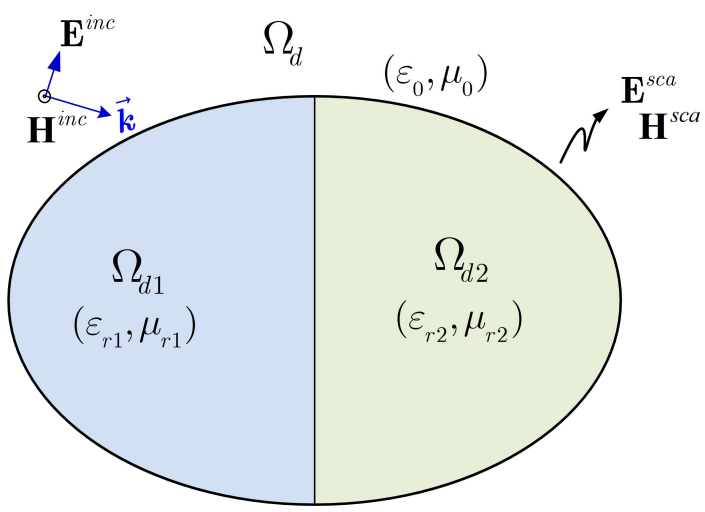

Fig. 1. EM scattering from a piecewise homogeneous object illuminated by a plane wave.

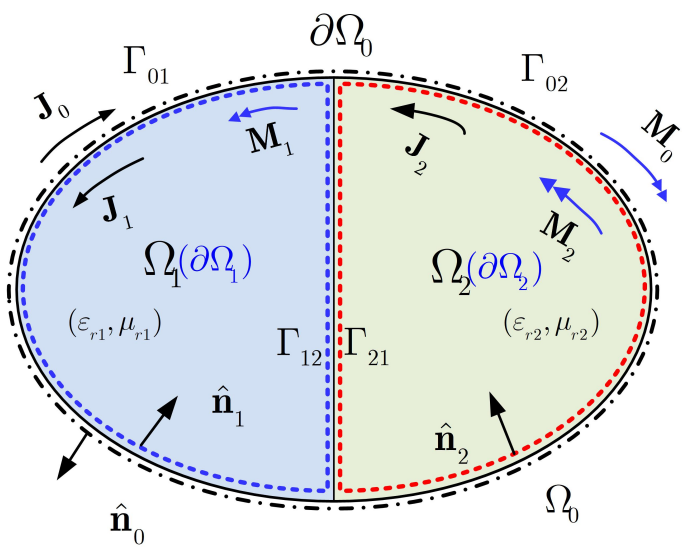

Fig. 2. The notations used in LCMT-DDM.

regions, the exterior region $\Omega_{0}$ with boundary $\partial \Omega_{0}$, and the interior regions $\Omega_{1}$ and $\Omega_{2}$ with boundaries $\partial \Omega_{1}$ and $\partial \Omega_{2}$, respectively. The outward unit normal of $\partial \Omega_{m}$ is $\hat{\mathbf{n}}_{m}(m=$ $0,1,2)$. The exterior boundary satisfies $\partial \Omega_{0}=\Gamma_{01} \cup \Gamma_{02}$, where $\Gamma_{01}=\partial \Omega_{0} \cap \partial \Omega_{1}, \Gamma_{02}=\partial \Omega_{0} \cap \partial \Omega_{2}$. The interior boundaries satisfy $\partial \Omega_{1}=\Gamma_{10} \cup \Gamma_{12}, \partial \Omega_{2}=\Gamma_{20} \cup \Gamma_{21}$, where $\Gamma_{10}=\partial \Omega_{1} \cap \partial \Omega_{0}, \Gamma_{12}=\partial \Omega_{1} \cap \partial \Omega_{2}, \Gamma_{20}=\partial \Omega_{2} \cap \partial \Omega_{0}$, $\Gamma_{21}=\partial \Omega_{2} \cap \partial \Omega_{1}$.

According to the Stratton-Chu representation formula, the scattering fields can be expressed as,

$$
\left[\begin{array}{c}
\mathbf{E}_{m}^{s c a} \\
\eta_{m} \mathbf{H}_{m}^{s c a}
\end{array}\right]=\left[\begin{array}{cc}
\eta_{m} \mathcal{L}_{m} & -\eta_{0} \mathcal{K}_{m} \\
\eta_{m} \mathcal{K}_{m} & \eta_{0} \mathcal{L}_{m}
\end{array}\right]\left[\begin{array}{c}
\mathbf{J}_{m} \\
\mathbf{M}_{m}
\end{array}\right]
$$

where the operators $\mathcal{L}_{m}$ and $\mathcal{K}_{m}$ in $\Omega_{m}$ can be defined as

$$
\begin{gathered}
\mathcal{L}_{m}\left(\mathbf{X}\left(\mathbf{r}^{\prime}\right) ; \partial \Omega_{m}\right)= \\
-j k_{m} \int_{\partial \Omega_{m}}\left[\mathbf{I}+\frac{1}{k_{m}^{2}} \nabla \nabla \cdot\right] \mathbf{X}\left(\mathbf{r}^{\prime}\right) G_{m}\left(\mathbf{r}, \mathbf{r}^{\prime}\right) d \mathbf{r}^{\prime} \\
\mathcal{K}_{m}\left(\mathbf{X}\left(\mathbf{r}^{\prime}\right) ; \partial \Omega_{m}\right)= \\
\int_{\partial \Omega_{m}} \nabla G_{m}\left(\mathbf{r}, \mathbf{r}^{\prime}\right) \times \mathbf{X}\left(\mathbf{r}^{\prime}\right) d \mathbf{r}^{\prime} .
\end{gathered}
$$

Here, the time convention is assumed as $e^{j \omega t}$, so that the Green's function for $\Omega_{m}$ can be written as $G_{m}\left(\mathbf{r}, \mathbf{r}^{\prime}\right)=$ $\frac{e^{-j k_{m}\left|\mathbf{r}-\mathbf{r}^{\prime}\right|}}{4 \pi\left|\mathbf{r}-\mathbf{r}^{\prime}\right|}$, where $k_{m}=k_{0} \sqrt{\mu_{r m} \varepsilon_{r m}}$ is the wavenumber in $\Omega_{m}, k_{0}=\omega \sqrt{\mu_{0} \varepsilon_{0}}$ is the free-space wavenumber, $\omega=2 \pi f$ 
is the angular frequency, and the principle value of $\mathcal{K}_{m}$ is defined as $\overline{\mathcal{K}}_{m}$. The surface tangential trace operator $\pi_{\tau}^{(m)}$ and the twisted surface tangential trace operator $\pi_{\times}^{(m)}$ on the boundary $\partial \Omega_{m}$ are introduced as following,

$$
\begin{aligned}
& \pi_{\tau}^{(m)}(\mathbf{u}):=\hat{\mathbf{n}}_{m} \times\left.\left(\mathbf{u} \times \hat{\mathbf{n}}_{m}\right)\right|_{\partial \Omega_{m}} . \\
& \pi_{\times}^{(m)}(\mathbf{u}):=\hat{\mathbf{n}}_{m} \times\left.\mathbf{u}\right|_{\partial \Omega_{m}} .
\end{aligned}
$$

If we assume there are virtual gaps between $\Omega_{0}, \Omega_{1}$, and $\Omega_{2}$, the original boundary value problem (BVP) of the while model can be cast into a set of BVPs,

$$
\begin{aligned}
& \nabla \times \nabla \times \mathbf{E}_{0}-k_{0}^{2} \mathbf{E}_{0}=0 \quad \text { in } \Omega_{0} \\
& \nabla \times \mu_{r 1}^{-1} \nabla \times \mathbf{E}_{1}-k_{0}^{2} \varepsilon_{r 1} \mathbf{E}_{1}=0 \quad \text { in } \Omega_{1} \\
& \nabla \times \mu_{r 2}^{-1} \nabla \times \mathbf{E}_{2}-k_{0}^{2} \varepsilon_{r 2} \mathbf{E}_{2}=0 \quad \text { in } \Omega_{2} \\
& \pi_{\tau}^{(0)}\left(\mathbf{E}_{0}\right)=\pi_{\tau}^{(1)}\left(\mathbf{E}_{1}\right) \quad \text { on } \Gamma_{01} \\
& \pi_{\tau}^{(0)}\left(\mathbf{E}_{0}\right)=\pi_{\tau}^{(2)}\left(\mathbf{E}_{2}\right) \quad \text { on } \Gamma_{02} \\
& \pi_{\times}^{(0)}\left(\nabla \times \mathbf{E}_{0}\right)=-\pi_{\times}^{(1)}\left(\frac{1}{\mu_{r 1}} \nabla \times \mathbf{E}_{1}\right) \quad \text { on } \Gamma_{01} \\
& \pi_{\times}^{(0)}\left(\nabla \times \mathbf{E}_{0}\right)=-\pi_{\times}^{(2)}\left(\frac{1}{\mu_{r 2}} \nabla \times \mathbf{E}_{2}\right) \quad \text { on } \Gamma_{02} \\
& \pi_{\tau}^{(1)}\left(\mathbf{E}_{1}\right)=\pi_{\tau}^{(2)}\left(\mathbf{E}_{2}\right) \quad \text { on } \Gamma_{12} \\
& \pi_{\times}^{(1)}\left(\frac{1}{\mu_{r 1}} \nabla \times \mathbf{E}_{1}\right)=-\pi_{\times}^{(2)}\left(\frac{1}{\mu_{r 2}} \nabla \times \mathbf{E}_{2}\right) \quad \text { on } \Gamma \\
& \lim _{|\mathbf{r}| \rightarrow \infty}|\mathbf{r}|\left(\nabla \times \mathbf{E}^{\text {sca }}-\jmath k_{0} \hat{\mathbf{r}} \times \mathbf{E}^{\text {sca }}\right)=0
\end{aligned}
$$

where (13) is the Silver-Müller radiation condition for the scattered field in the exterior region $\Omega_{0}$. The total electric fields in $\Omega_{0}, \Omega_{1}$, and $\Omega_{2}$ are denoted as $\mathbf{E}_{0}, \mathbf{E}_{1}$, and $\mathbf{E}_{2}$, which can be obtained by solving (4)-(6). By invoking the equivalence principle for each sub-region $\Omega_{m}$, the electric field integral equation $\left(\mathrm{EFIE}_{m}\right)$ and mangetic field integral equation $\left(\mathrm{MFIE}_{m}\right)$ can be derived as

$$
\begin{aligned}
& \left\{\begin{array}{l}
\frac{\eta_{0}}{2} \pi_{\times}^{(0)} \mathbf{M}_{0}(\mathbf{r})-\pi_{\tau}^{(0)}\left(\eta_{0} \mathcal{L}_{0}\left(\mathbf{J}_{0}\left(\mathbf{r}^{\prime}\right) ; \partial \Omega_{0}\right)\right) \\
+\pi_{\tau}^{(0)}\left(\eta_{0} \overline{\mathcal{K}}_{0}\left(\mathbf{M}_{0}\left(\mathbf{r}^{\prime}\right) ; \partial \Omega_{0}\right)\right)=\pi_{\tau}^{(0)}\left(\mathbf{E}^{i n c}(\mathbf{r})\right) \\
-\frac{\eta_{0}}{2} \pi_{\times}^{(0)} \mathbf{J}_{0}(\mathbf{r})-\pi_{\tau}^{(0)}\left(\eta_{0} \mathcal{L}_{0}\left(\mathbf{M}_{0}\left(\mathbf{r}^{\prime}\right) ; \partial \Omega_{0}\right)\right) \\
-\pi_{\tau}^{(0)}\left(\eta_{0} \overline{\mathcal{K}}_{0}\left(\mathbf{J}_{0}\left(\mathbf{r}^{\prime}\right) ; \partial \Omega_{0}\right)\right)=\pi_{\tau}^{(0)}\left(\eta_{0} \mathbf{H}^{i n c}(\mathbf{r})\right)
\end{array}\right. \\
& \left\{\begin{array}{l}
\frac{\eta_{0}}{2} \pi_{\times}^{(m)} \mathbf{M}_{m}(\mathbf{r})-\pi_{\tau}^{(m)}\left(\eta_{m} \mathcal{L}_{m}\left(\mathbf{J}_{m}\left(\mathbf{r}^{\prime}\right) ; \partial \Omega_{m}\right)\right) \\
+\pi_{\tau}^{(m)}\left(\eta_{0} \overline{\mathcal{K}}_{m}\left(\mathbf{M}_{m}\left(\mathbf{r}^{\prime}\right) ; \partial \Omega_{m}\right)\right)=0 \\
-\frac{\eta_{m}}{2} \pi_{\times}^{(m)} \mathbf{J}_{m}(\mathbf{r})-\pi_{\tau}^{(m)}\left(\eta_{0} \mathcal{L}_{m}\left(\mathbf{M}_{m}\left(\mathbf{r}^{\prime}\right) ; \partial \Omega_{m}\right)\right) \\
-\pi_{\tau}^{(m)}\left(\eta_{m} \overline{\mathcal{K}}_{m}\left(\mathbf{J}_{m}\left(\mathbf{r}^{\prime}\right) ; \partial \Omega_{m}\right)\right)=0
\end{array}\right.
\end{aligned}
$$

where (14) is for the exterior sub-domain and (15) is for the interior sub-domains $(m=1,2)$. To make sure that the solution of the decomposed problem is equivalent to that of the original one, the boundary conditions in (7)-(12) should be properly enforced. The following RTCs, which are equivalent to these boundary conditions, are used in LCMT-DDM:

$\left\{\begin{array}{l}\eta_{r m} \mathbf{J}_{m}-\hat{\mathbf{n}}_{m} \times \mathbf{M}_{m}+\eta_{r m} \mathbf{J}_{n}+\hat{\mathbf{n}}_{n} \times \mathbf{M}_{n}=0 \\ \eta_{r m} \hat{\mathbf{n}}_{m} \times \mathbf{J}_{m}+\mathbf{M}_{m}-\eta_{r m} \hat{\mathbf{n}}_{n} \times \mathbf{J}_{n}+\mathbf{M}_{n}=0\end{array}, \mathbf{r} \in \Gamma_{m n}\right.$ where $m, n=0,1,2, m \neq n, \eta_{r m}=\eta_{m} / \eta_{0}$ and $\Gamma_{m n}$ means the touching face between adjacent sub-domain $m$ and subdomain $n$.

By linearly combining the IEs and RTCs as $(14)+\frac{1}{2} \eta_{0}(16)$ and $(15)+\frac{1}{2} \eta_{0}(16)$, the resultant equations can be expressed as

$$
\begin{aligned}
& \frac{\eta_{0}}{2} \mathbf{J}_{0}(\mathbf{r})-\eta_{0} \pi_{\tau}^{(0)}\left(\mathcal{L}_{0}\left(\mathbf{J}_{0}\left(\mathbf{r}^{\prime}\right) ; \partial \Omega_{0}\right)-\overline{\mathcal{K}}_{0}\left(\mathbf{M}_{0}\left(\mathbf{r}^{\prime}\right) ; \partial \Omega_{0}\right)\right) \\
& +\frac{\eta_{0}}{2}\left\{\mathbf{J}_{n}+\hat{\mathbf{n}}_{n} \times \mathbf{M}_{n}\right\}=\pi_{\tau}^{(0)}\left(\mathbf{E}^{i n c}(\mathbf{r})\right), \mathbf{r} \in \Gamma_{0 n} \\
& \frac{\eta_{0}}{2} \mathbf{M}_{0}(\mathbf{r})-\eta_{0} \pi_{\tau}^{(0)}\left(\mathcal{L}_{0}\left(\mathbf{M}_{0}\left(\mathbf{r}^{\prime}\right) ; \partial \Omega_{0}\right)+\overline{\mathcal{K}}_{0}\left(\mathbf{J}_{0}\left(\mathbf{r}^{\prime}\right) ; \partial \Omega_{0}\right)\right) \\
& +\frac{\eta_{0}}{2}\left\{\mathbf{M}_{n}-\hat{\mathbf{n}}_{n} \times \mathbf{J}_{n}\right\}=\eta_{0} \pi_{\tau}^{(0)}\left(\mathbf{H}^{i n c}(\mathbf{r})\right), \mathbf{r} \in \Gamma_{0 n} \\
& -\pi_{\tau}^{(m)}\left(\eta_{m} \mathcal{L}_{m}\left(\mathbf{J}_{m}\left(\mathbf{r}^{\prime}\right) ; \partial \Omega_{m}\right)-\eta_{0} \overline{\mathcal{K}}_{m}\left(\mathbf{M}_{m}\left(\mathbf{r}^{\prime}\right) ; \partial \Omega_{m}\right)\right) \\
& +\frac{\eta_{m}}{2} \mathbf{J}_{m}(\mathbf{r})+\frac{1}{2}\left\{\eta_{m} \mathbf{J}_{n}+\eta_{0} \hat{\mathbf{n}}_{n} \times \mathbf{M}_{n}\right\}=0, \mathbf{r} \in \Gamma_{m n} \\
& -\pi_{\tau}^{(m)}\left(\eta_{0} \mathcal{L}_{m}\left(\mathbf{M}_{m}\left(\mathbf{r}^{\prime}\right) ; \partial \Omega_{m}\right)+\eta_{m} \overline{\mathcal{K}}_{m}\left(\mathbf{J}_{m}\left(\mathbf{r}^{\prime}\right) ; \partial \Omega_{m}\right)\right) \\
& +\frac{\eta_{0}}{2} \mathbf{M}_{m}(\mathbf{r})+\frac{1}{2}\left\{\eta_{0} \mathbf{M}_{n}-\eta_{m} \hat{\mathbf{n}}_{n} \times \mathbf{J}_{n}\right\}=0, \mathbf{r} \in \Gamma_{m n}
\end{aligned}
$$

where $n=1,2$ in (17), and $m, n$ satisfy $\{m=1,2\} \cap\{n=$ $0,1,2\} \cap\{m \neq n\}$ in (18).

\section{B. Matrices Formulation}

Equations (17) and (18) can be discretized by using the Rao-Wilton-Glisson (RWG) basis function and Galerkin test [27]. The resultant linear system can then be written as:

$$
\left[\begin{array}{ccc}
\mathbf{A}_{0} & \mathbf{M}_{01} & \mathbf{M}_{02} \\
\mathbf{M}_{10} & \mathbf{A}_{1} & \mathbf{M}_{12} \\
\mathbf{M}_{20} & \mathbf{M}_{21} & \mathbf{A}_{2}
\end{array}\right]\left[\begin{array}{l}
\mathbf{I}_{0} \\
\mathbf{I}_{1} \\
\mathbf{I}_{2}
\end{array}\right]=\left[\begin{array}{c}
\mathbf{V}^{0} \\
\mathbf{0} \\
\mathbf{0}
\end{array}\right]
$$

where $\mathbf{A}_{n}$ is either the self-interaction matrix for the exterior sub-domain $(n=0)$ or the interior sub-domains $(n=1,2)$, and $\mathbf{M}_{m n}$ is the mutual-interaction matrix between any two sub-domains. Due to the local coupling nature of LCMTDDM, $\mathbf{M}_{m n}$ is either a sparse matrix for touching subdomains or a zero matrix for non-touching sub-domains. Moreover, $\mathbf{I}_{n}$ and $\mathbf{V}^{0}$ can be expressed as $\mathbf{I}_{n}=\left(\mathbf{J}_{n}, \mathbf{M}_{n}\right)$ and $\mathbf{V}^{0}=\left(\mathbf{V}_{j}^{0}, \mathbf{V}_{m}^{0}\right)$, and $\mathbf{A}_{n}$ can further be written as:

$$
\mathbf{A}_{n}=\left[\begin{array}{cc}
\mathbf{A}_{j j}^{n} & \mathbf{A}_{j m}^{n} \\
\mathbf{A}_{m j}^{n} & \mathbf{A}_{m m}^{n}
\end{array}\right] .
$$

The detailed expressions of these sub-matrices can be derived as:

$$
\begin{aligned}
& \mathbf{A}_{j j}^{n}=\left\langle\mathbf{f}_{n}(\mathbf{r}), \frac{\eta_{n}}{2} \mathbf{f}_{n}(\mathbf{r})-\eta_{n} \pi_{\tau}^{(n)} \mathcal{L}_{n}\left(\mathbf{f}_{n}\left(\mathbf{r}^{\prime}\right) ; \partial \Omega_{n}\right)\right\rangle_{\partial \Omega_{n}} \\
& \mathbf{A}_{j m}^{n}=\left\langle\mathbf{f}_{n}(\mathbf{r}), \eta_{0} \pi_{\tau}^{(n)} \overline{\mathcal{K}}_{n}\left(\mathbf{m}_{n}\left(\mathbf{r}^{\prime}\right) ; \partial \Omega_{n}\right)\right\rangle_{\partial \Omega_{n}} \\
& \mathbf{A}_{m j}^{n}=\left\langle\mathbf{m}_{n}(\mathbf{r}),-\eta_{n} \pi_{\tau}^{(n)} \overline{\mathcal{K}}_{n}\left(\mathbf{f}_{n}\left(\mathbf{r}^{\prime}\right) ; \partial \Omega_{n}\right)\right\rangle_{\partial \Omega_{n}} \\
& \mathbf{A}_{m m}^{n}=\left\langle\mathbf{m}_{n}(\mathbf{r}), \frac{\eta_{0}}{2} \mathbf{m}_{n}(\mathbf{r})-\eta_{0} \pi_{\tau}^{(n)} \mathcal{L}_{n}\left(\mathbf{m}_{n}\left(\mathbf{r}^{\prime}\right) ; \partial \Omega_{n}\right)\right\rangle_{\partial \Omega_{n}}
\end{aligned}
$$

where $\mathbf{f}_{n}$ and $\mathbf{m}_{n}$ denote as the basis function for the electric and magnetic currents $\mathbf{J}_{n}$ and $\mathbf{M}_{n}$, respectively, and the inner 
product of two complex-valued vector functions $\mathbf{u}, \mathbf{v}$ is defined as

$$
\langle\mathbf{u}, \mathbf{v}\rangle_{\Gamma}=\int_{\Gamma}(\mathbf{u} \cdot \mathbf{v}) d \Gamma
$$

Looking at the expressions of the diagonal blocks $\mathbf{A}_{j j}^{n}$ and $\mathbf{A}_{m m}^{n}$ in (21), one can see that their entries consist of tested contributions from EFIE and the residue term of MFIE (same structure as CFIE). This makes $\mathbf{A}_{n}$ a diagonally dominant matrix and, therefore, its nearfield component (which also includes the diagonal entries) is used to precondition the original linear system matrix as described in Section II-C. The sparse matrix $\mathbf{M}_{m n}$ and the right-hand-side (RHS) vectors $\mathbf{V}_{j}^{0}, \mathbf{V}_{m}^{0}$ can further be expressed as

$\mathbf{M}_{m n}=\left[\begin{array}{cc}\frac{\eta_{m}}{2}\left\langle\mathbf{f}_{m}, \mathbf{f}_{n}\right\rangle_{\Gamma_{m n}} & \frac{\eta_{0}}{2}\left\langle\mathbf{f}_{m}, \hat{\mathbf{n}}_{n} \times \mathbf{m}_{n}\right\rangle_{\Gamma_{m n}} \\ -\frac{\eta_{0}}{2}\left\langle\mathbf{m}_{m}, \hat{\mathbf{n}}_{n} \times \mathbf{f}_{n}\right\rangle_{\Gamma_{m n}} & \frac{\eta_{m}}{2}\left\langle\mathbf{m}_{m}, \mathbf{m}_{n}\right\rangle_{\Gamma_{m n}}\end{array}\right]$

and

$$
\begin{aligned}
\mathbf{V}_{j}^{0} & =\left\langle\mathbf{f}_{j}^{o}(\mathbf{r}), \pi_{\tau}^{(0)}\left(\mathbf{E}^{i n c}(\mathbf{r})\right)\right\rangle_{\partial \Omega_{0}} \\
\mathbf{V}_{m}^{0} & =\left\langle\mathbf{m}_{d}^{o}(\mathbf{r}), \eta_{o} \pi_{\tau}^{(0)}\left(\mathbf{H}^{i n c}(\mathbf{r})\right)\right\rangle_{\partial \Omega_{0}} .
\end{aligned}
$$

To further show the local coupling nature and the resultant sparse matrix of LCMT-DDM, we take the problem in Fig. 3 for example. In Fig. 3, the original multilayered dielectric object is decomposed into 5 sub-domains, and the system matrix can be written in the form of (25). In (25), $\mathbf{A}_{i i}(i=0,1, \ldots 4)$ is the dense self-coupling matrix for each sub-domain, $\mathbf{M}_{i j}$ is the sparse local coupling matrix. From Fig 3, it can be observed that $\Omega_{1}$ does not touch $\Omega_{3}, \Omega_{4}$ and $\Omega_{2}$ does not touch $\Omega_{4}$, therefore the mutual coupling matrices for these sub-domains are all zero, as shown in (25). Obviously, for a multilayered structure, the system matrix is even more sparse.

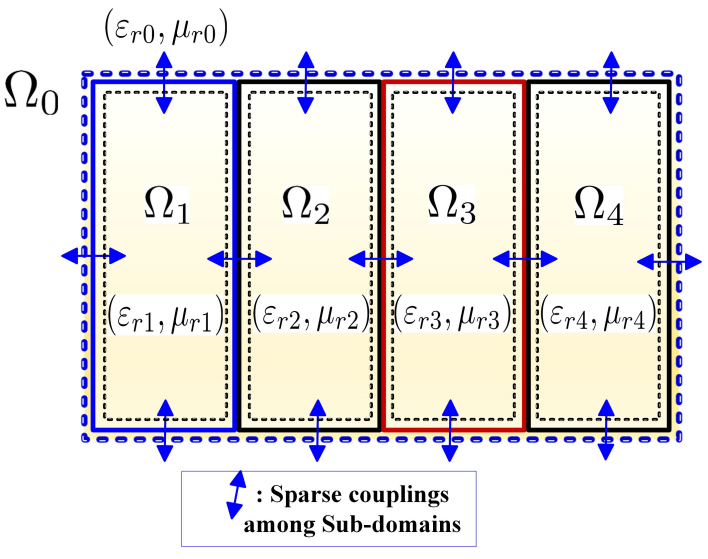

Fig. 3. Domain decomposition notation of five sub-regions. Arrows stand for the coupling between different sub-regions, which only exist between neighboring regions.

$$
\mathcal{A}=\left[\begin{array}{ccccc}
\mathbf{A}_{00} & \mathbf{M}_{01} & \mathbf{M}_{02} & \mathbf{M}_{03} & \mathbf{M}_{04} \\
\mathbf{M}_{10} & \mathbf{A}_{11} & \mathbf{M}_{12} & 0 & 0 \\
\mathbf{M}_{20} & \mathbf{M}_{21} & \mathbf{A}_{22} & \mathbf{A}_{23} & 0 \\
\mathbf{M}_{30} & 0 & \mathbf{A}_{32} & \mathbf{A}_{33} & \mathbf{M}_{34} \\
\mathbf{M}_{40} & 0 & 0 & \mathbf{M}_{43} & \mathbf{A}_{44}
\end{array}\right]
$$

\section{Preconditioning Technique}

In the proposed LCMT-DDM, the FGMRES method, which is an inner-outer iteration scheme, is used to solve the preconditioned system,

$$
\mathcal{A} \mathcal{P}^{-1} \mathcal{P} \mathbf{x}=\mathbf{b}
$$

where $\mathcal{A}$ is the matrix of the linear system shown in (19), and $\mathcal{P}^{-1}$ is the preconditioner.

In this work, $\mathcal{P}$ is constructed as:

$$
\mathcal{P}=\left[\begin{array}{ccc}
\mathbf{A}_{\text {nea } 0} & \mathbf{0} & \mathbf{0} \\
\mathbf{M}_{10} & \mathbf{A}_{\text {nea } 1} & \mathbf{0} \\
\mathbf{M}_{20} & \mathbf{M}_{12} & \mathbf{A}_{\text {nea } 2}
\end{array}\right]
$$

where $\mathbf{A}_{\text {neai }}(i=0,1,2)$ is the near-group coupling matrix in MLMFA for each sub-domain. Then the inverse of $\mathcal{P}$ has the form of

$$
\mathcal{P}^{-1}=\left[\begin{array}{ccc}
\mathbf{A}_{\text {nea } 0}^{-1} & \mathbf{0} & \mathbf{0} \\
\mathbf{B} & \mathbf{A}_{\text {nea } 1}^{-1} & \mathbf{0} \\
\mathbf{D} & \mathbf{C} & \mathbf{A}_{\text {nea } 2}^{-1}
\end{array}\right]
$$

where $\mathbf{B}, \mathbf{C}, \mathbf{D}$ can be explicitly calculated as

$$
\begin{gathered}
\mathbf{B}=-\mathbf{A}_{\text {nea } 1}^{-1} \mathbf{M}_{10} \mathbf{A}_{\text {nea } 0}^{-1} \\
\mathbf{C}=-\mathbf{A}_{\text {nea } 2}^{-1} \mathbf{M}_{21} \mathbf{A}_{\text {nea } 1}^{-1} \\
\mathbf{D}=-\mathbf{A}_{\text {nea } 2}^{-1}\left(\mathbf{M}_{20}-\mathbf{M}_{21} \mathbf{A}_{\text {nea } 1}^{-1} \mathbf{M}_{10}\right) \mathbf{A}_{\text {nea } 0}^{-1}
\end{gathered}
$$

The procedure of the inner-outer iteration in FGMRES is shown in Algorithm 1. Compared with the traditional GMRES method, the only difference in FGMRES is that the preconditioned vector $\mathbf{z}_{j}=\mathcal{P}^{-1} \mathbf{v}_{j}$ needs to be computed (see Algorithm 1). In order to obtain the preconditioner $\mathcal{P}^{-1}$ in (28), $\mathbf{A}_{\text {neai }}^{-1}(i=0,1,2)$ should be calculated. In FGMRES, this can be accomplished by an inner iteration. Since $\mathbf{A}_{\text {neai }}$ in our method is diagonally dominant, the preconditioned vector $\mathbf{z}_{j}$ can be efficiently calculated in the inner iteration without extra memory consumption.

To better explain the procedure, the indices of the matrices will be started from 1 instead of 0 . Moreover, we denote $\mathbf{P}_{1,1}=\mathbf{A}_{\text {nea } 0}, \mathbf{P}_{2,2}=\mathbf{A}_{\text {nea } 1}$, and $\mathbf{P}_{3,3}=\mathbf{A}_{\text {nea } 2}$. Then $\mathcal{P}$ in (27) can be rewritten as

$$
\mathcal{P}=\mathcal{P}_{3,3}=\left[\begin{array}{ccc}
\mathbf{P}_{1,1} & 0 & 0 \\
\mathbf{M}_{2,1} & \mathbf{P}_{2,2} & 0 \\
\mathbf{M}_{3,1} & \mathbf{M}_{3,2} & \mathbf{P}_{3,3}
\end{array}\right] .
$$

If we denote the upper-left $1 \times 1$ block as

$$
\mathcal{P}_{1,1}=\left[\mathbf{P}_{1,1}\right],
$$

then the upper-left $2 \times 2$ block can be denoted as

$$
\mathcal{P}_{2,2}=\left[\begin{array}{cc}
\mathcal{P}_{1,1} & 0 \\
\mathbf{M}_{2,1} & \mathbf{P}_{2,2}
\end{array}\right] .
$$

Similarly, $\mathcal{P}_{3,3}$ can be expressed as,

$$
\mathcal{P}_{3,3}=\left[\begin{array}{ll}
\mathcal{P}_{2,2} & \mathbf{0} \\
\mathbf{M}_{3,1 \rightarrow 2} & \mathbf{P}_{3,3}
\end{array}\right] .
$$

where $\mathbf{M}_{3,1 \rightarrow 2}$ stands for $\left[\mathbf{M}_{3,1}, \mathbf{M}_{3,2}\right]$. Apparently, this notation leads to a recursive formulation. Generally, when the problem is decomposed into $L$ regions, $\mathcal{P}$ can be written as

$$
\mathcal{P}=\mathcal{P}_{L, L}=\left[\begin{array}{ll}
\mathcal{P}_{L-1, L-1} & \mathbf{0} \\
\mathbf{M}_{L, 1 \rightarrow L-1} & \mathbf{P}_{L, L}
\end{array}\right] .
$$


Hence, the preconditioner $\mathcal{P}^{-1}$ can be calculated as

$$
\mathcal{P}^{-1}=\mathcal{P}_{L, L}^{-1}=\left[\begin{array}{cc}
\mathcal{P}_{L-1, L-1}^{-1} & 0 \\
-\mathbf{P}_{L, L}^{-1} \mathbf{M}_{L, 1 \rightarrow L-1} \mathcal{P}_{L-1, L-1}^{-1} & \mathbf{P}_{L, L}^{-1}
\end{array}\right]
$$

Consequently, the multiplication of $\mathcal{P}^{-1}$ and the vector $\mathbf{X}_{L}=$ $\left(\mathbf{X}_{L-1} ; \mathbf{x}_{L}^{\mathrm{T}}\right)=\left(\mathbf{x}_{1}, \mathbf{x}_{2}, \mathbf{x}_{3} \cdots \mathbf{x}_{L}\right)^{\mathrm{T}}$ can be expressed as:

$$
\begin{aligned}
& \mathbf{Y}_{L}=\mathcal{P}^{-1} \mathbf{X}_{L}=\mathcal{P}_{L, L}^{-1}\left[\begin{array}{c}
\mathbf{X}_{L-1} \\
\mathbf{x}_{L}
\end{array}\right] \\
& =\left[\begin{array}{cc}
\mathcal{P}_{L-1, L-1}^{-1} & 0 \\
-\mathbf{P}_{L, L}^{-1} \mathbf{M}_{L, 1 \rightarrow L-1} \mathcal{P}_{L-1, L-1}^{-1} & \mathbf{P}_{L, L}^{-1}
\end{array}\right]\left[\begin{array}{c}
\mathbf{X}_{L-1} \\
\mathbf{x}_{L}
\end{array}\right] \\
& =\left[\begin{array}{c}
\mathbf{Y}_{L-1} \\
\mathbf{P}_{L, L}^{-1}\left(-\left[\mathbf{M}_{L, 1} \mathbf{y}_{1}+\cdots+\mathbf{M}_{L, L-1} \mathbf{y}_{L-1}\right]+\mathbf{x}_{L}\right)
\end{array}\right]
\end{aligned}
$$

where $\mathbf{Y}_{L}=\left(\mathbf{Y}_{L-1} ; \mathbf{y}_{L}^{\mathrm{T}}\right)=\left(\mathbf{y}_{1}, \mathbf{y}_{2}, \mathbf{y}_{3} \cdots \mathbf{y}_{L}\right)^{\mathrm{T}}$. It is obvious that $\mathcal{P}^{-1} \mathbf{X}_{L}$ can be calculated recursively. In the computation, we only need to iteratively solve a sequence of linear systems as

$$
\mathbf{P}_{l, l} \mathbf{y}_{l}=\mathbf{x}_{l} \quad(l=1, \cdots \mathrm{L})
$$

to finally obtain the vector $\mathbf{Y}_{L}$. Because of the sparse nature and favorable iterative convergence of $\mathbf{P}_{l, l}$ (self- and neargroup interaction of CFIE-like matrices in MLFMA), the preconditioner can be efficiently applied within the inner iterations of FGMRES (which is a GMRES process [18]). The details of the FGMRES procedure can be found in [23].

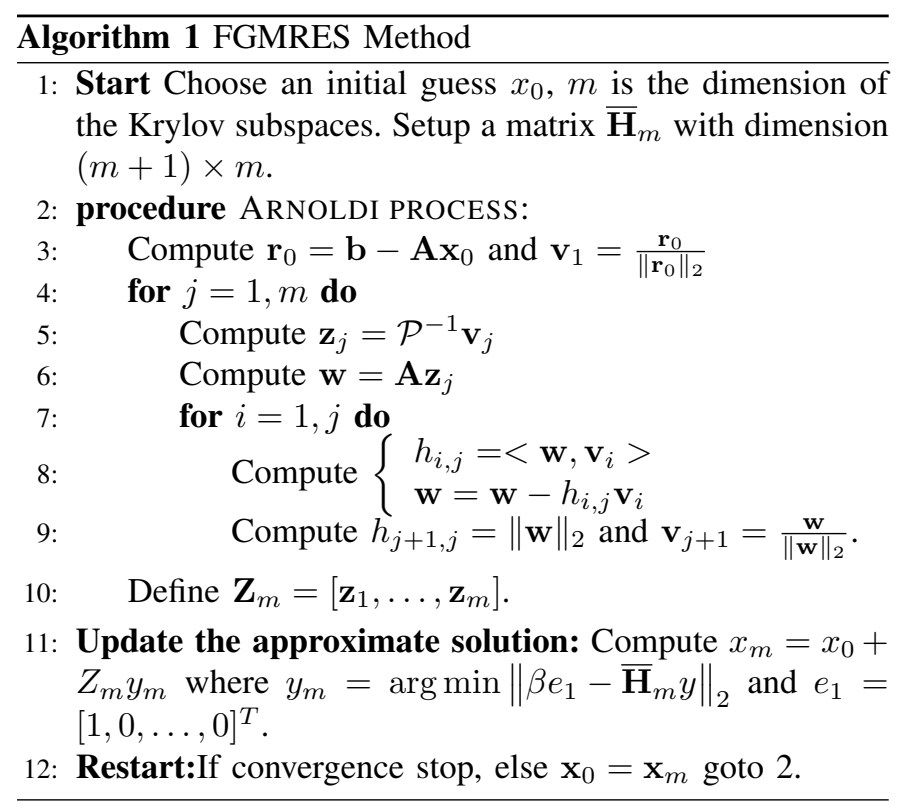

\section{NUMERICAL RESUlTS}

In this section, the accuracy and capability of the proposed LCMT-DDM are validated via several numerical examples. A homogeneous dielectric sphere and a two-layer sphere are first studied to show the high accuracy of this method. The effect of the inner-iteration tolerance on the outer iteration and the overall solution accuracy are also investigated. Then two multilayered dielectric objects are further studied to demonstrate the superior performance of this method for more complicated objects, including accuracy, iterative convergence, and memory consumption.

\section{A. Validation of LCMT-DDM}

In the first numerical example, the EM scattering from a homogeneous dielectric sphere is investigated to demonstrate the accuracy of LCMT-DDM. The radius of the sphere is $0.5 \mathrm{~m}$, and the relative permittivity, permeability are $\varepsilon_{r 1}=$ $2.0, \mu_{r 1}=1.0$. The plane wave is incident from the $-z$ axis at $0.3 \mathrm{GHz}$. In LCMT-DDM, the original object is decomposed into three regions, the exterior region and two hemi-sphere interior regions. The exterior region is discretized with 800 triangular patches, and the interior regions are discretized with 644 and 688 triangular patches, respectively. The V-V and H$\mathrm{H}$ polarized bistatic radar cross section (RCS) of this dielectric sphere is calculated and shown in Fig. 4. For comparison, the analytical results from the Mie series are also shown. It can be seen that the results of the proposed method agree well with the Mie solution.

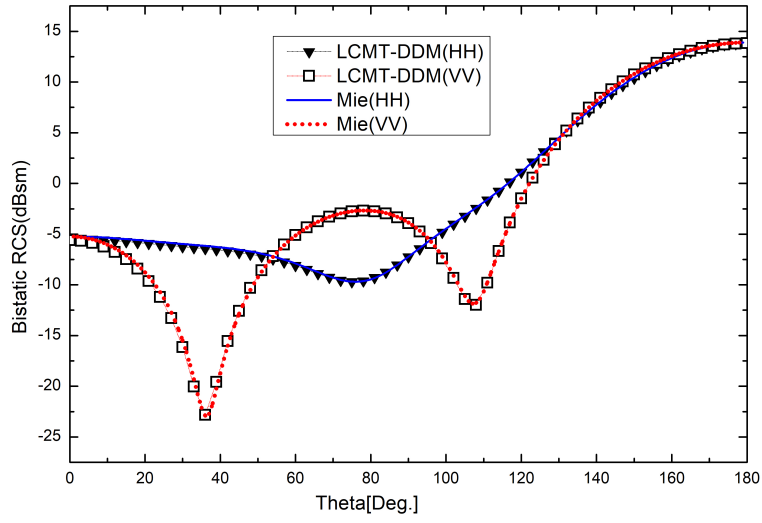

Fig. 4. The bistatic RCS of the dielectric sphere.

Next, we consider a two-layer dielectric sphere. It is composed of a dielectric sphere with radius of $0.5 \mathrm{~m}$ and a dielectric coating layer with thickness of $0.1 \mathrm{~m}$. The relative permittivity, permeability of the coating and sphere are $\varepsilon_{r 1}, \mu_{r 1}$ and $\varepsilon_{r 2}, \mu_{r 2}$, respectively. To further demonstrate the accuracy of LCMT-DDM, the root mean square error (RMSE) of RCS is investigated, which is defined as

$$
\text { RMSE }=\sqrt{\frac{1}{N} \sum_{i=1}^{N}\left|\sigma_{i}-\sigma_{i}^{M i e}\right|^{2}} .
$$

where $N$ is the total number of observation points, $\sigma$ is the RCS calculated from numerical methods, and $\sigma_{i}^{M i e}$ is obtained by the Mie series. The incidence of the plane wave and the working frequency are set the same as the previous case. For comparison, the traditional CRM is also implemented. Two material settings are used, i.e. $\varepsilon_{r 1}=9.0, \mu_{r 1}=1.0, \varepsilon_{r 2}=$ $8.0, \mu_{r 2}=1.0$ and $\varepsilon_{r 1}=3.0, \mu_{r 1}=1.0, \varepsilon_{r 2}=2.0, \mu_{r 2}=$ 1.0. In Fig. 5, RMSE of LCMT-DDM and CRM are computed and compared for different discretization densities. Here, fourpoint Gaussian quadrature rule is used in each triangle. It can 
be seen that the two methods have almost the same accuracy under the same discretization, and the error decreases at the same speed when the discretization density increases.

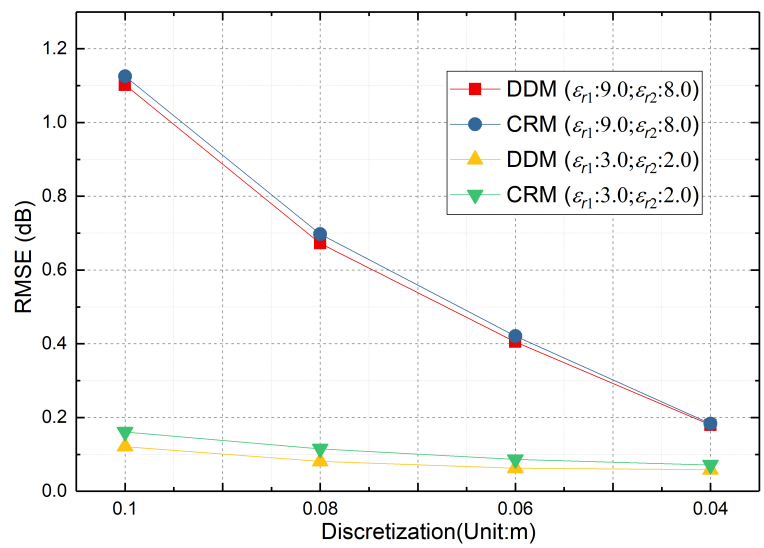

Fig. 5. The RMSE of bistatic RCS of the two-layer dielectric sphere.

We also examine the accuracy and the outer iteration count affected by the convergence tolerance of the inner iteration. The convergence tolerance of the outer iteration is fixed as 0.001. As shown in Table I, the outer iteration count does not change with respect to the inner convergence tolerances $(0.01$, $0.001,0.0001)$, and the variation of the RCS error is within $0.01 \mathrm{~dB}$. It reveals that the accuracy is mainly governed by the tolerance of the outer iteration. The inner iteration does not have a significant impact on the outer iteration and the final results.

TABLE I

THE RMSE AND OUTER ITERATION COUNT WITH DIFFERENT INNER TOL.

\begin{tabular}{cccc}
\hline \hline Inner tol. & 0.01 & 0.001 & 0.0001 \\
\hline RMSEs(dBsm) & 0.1409 & 0.1373 & 0.1354 \\
\hline Outer Num. & 29 & 29 & 29 \\
\hline \hline
\end{tabular}

\section{B. EM Scattering from Multilayered Dielectric Plate}

In the second numerical example, the EM scattering from a two-layer dielectric plate and a four-layer dielectric plate is investigated. The computation was performed on a personal computer with Intel Core i7-7700K @ 4.20GHz CPU. The geometry, dimension and material of the four-layer plate are shown in Fig. 6, and the two-layer model is just one half of the four-layer model. As shown in this figure, this multilayered plate consists of two alternately stacked slabs with different materials but same size.

The plane wave is incident from the $-z$ direction at $3 \mathrm{GHz}$. In LCMT-DDM, the two-layer and four-layer plates are decomposed into three and five regions respectively according to the material configuration. In the four-layer model, the number of triangles in each sub-regions of LCMT-DDM is, Region 0 : 72,392, Region 1 : 48,296, Region 2 : 48, 296, Region $_{3}$ : 48,296, and Region 4 : 48,296. The bistatic RCS results of the two models are shown in Fig. 7 and Fig. 8. For

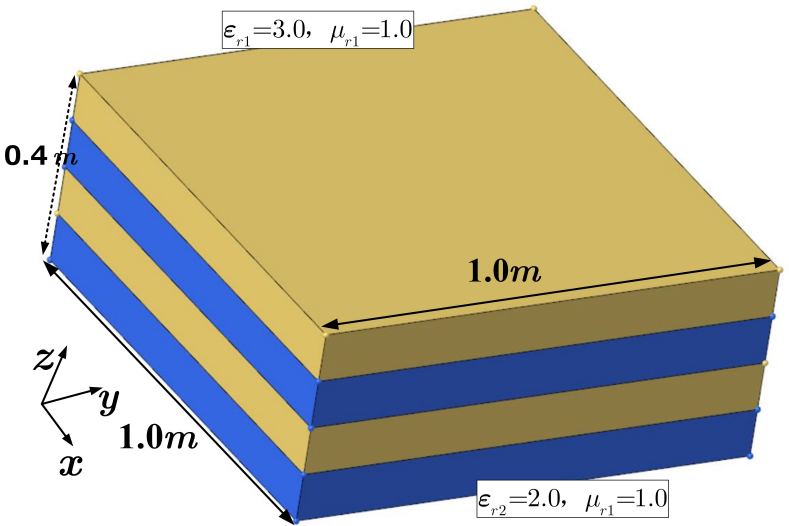

Fig. 6. The geometry of the four-layer dielectric plate.

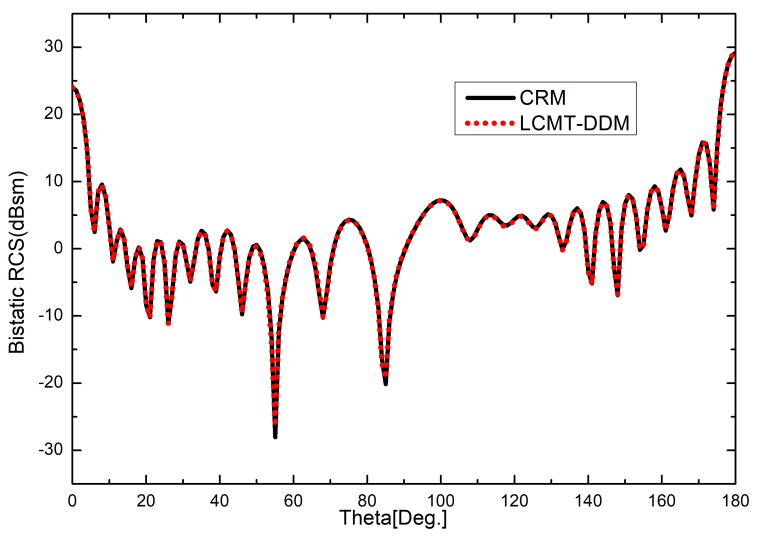

Fig. 7. The bistatic RCS of the two-layer dielectric plate.

comparison, the RCS calculated from CRM is also plotted. The discretization density of CRM is set as the same as that in LCMT-DDM for a fair comparison. It can be seen that the results of the two methods agree very well with each other.

The GMRES method utilized by CRM uses a tolerance of 0.001. The outer iteration convergence tolerance of FGMRES used by LCMT-DDM is also set to 0.001 . The inner iteration convergence tolerance of FGMRES is set to 0.01. The consumption of computational resources is shown in Table II and Table III for the two models. It can be seen that LCMT-DDM has an apparent advantage over the traditional CRM for both cases. Enforcing local coupling between different domains reduces the memory requirement of LCMT-DDM (compared to the traditional CRM method) since it makes the coupling matrices sparse (as opposed to dense matrices in CRM). The CPU time required by LCMT-DDM is also reduced. This is because the flexible preconditioner used in the inner iterations of FGMRES significantly increases the convergence rate.

\section{EM Scattering from a Multilayered Dielectric Radome}

In the third numerical example, the EM scattering from a three-layer dielectric radome is investigated. This computation was performed on a workstation with two 64-bit Intel Xeon 


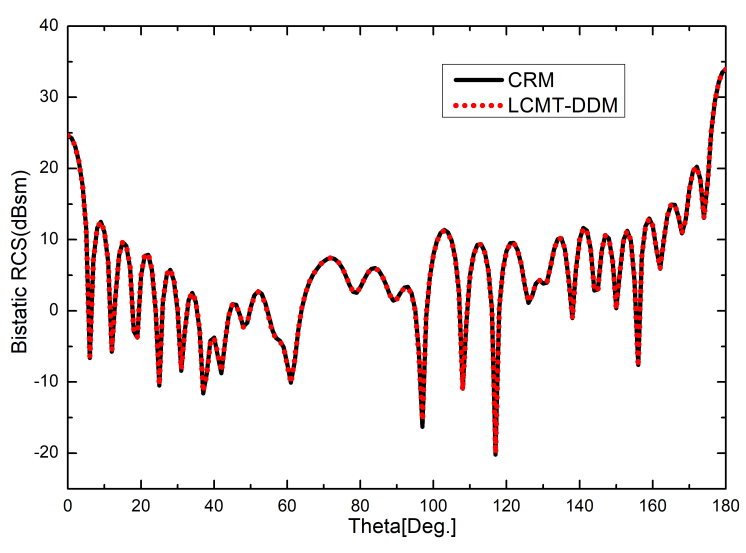

Fig. 8. The bistatic RCS of the four-layer dielectric plate.

TABLE II

COMPUTATIONAL STATISTICS OF THE TWO-LAYER PLATE

\begin{tabular}{cccc}
\hline \hline & Time & Men. & Iter. Num. \\
\hline LCMT-DDM: & 1,750 Sec. & 4.7GB & 83 \\
\hline CRM: & 5,652 Sec. & $7.0 \mathrm{~GB}$ & 637 \\
\hline \hline
\end{tabular}

TABLE III

COMPUTATIONAL STATISTICS OF THE FOUR-LAYER PLATE

\begin{tabular}{cccc}
\hline \hline & Time & Men. & Iter. Num. \\
\hline LCMT-DDM: & 3,976 Sec. & 7.9GB & 127 \\
\hline CRM: & 15,308 Sec. & $12.7 \mathrm{~GB}$ & 943 \\
\hline \hline
\end{tabular}

Gold $6148 @ 2.40 \mathrm{GHz}$ CPUs and run with twenty openMP parallel processes. The geometry and dimension of the radome are shown in Fig. 9. As shown in the figure, the radius of the air hemi-sphere is $r=584 \mathrm{~mm}$. The thickness of each layer is $d=18 \mathrm{~mm}$. The relative permittivity and relative permeability of the outermost layer and the innermost layer are both $\varepsilon_{r 1}=3.0, \mu_{r 1}=1.0$, and those of the middle layer are $\varepsilon_{r 2}=2.0, \mu_{r 2}=1.0$. The plane wave is incident from the $-z$ axis, and the frequency is $4.0 \mathrm{GHz}$. In LCMT-DDM, the radome is decomposed into four homogeneous sub-regions based on their material properties. The mesh size in each subregion is set to be $7.5 \mathrm{~mm}$, and the numbers of triangles in each sub-region are, Region R $_{0}$ 170,708, Region ${ }_{1}: 173,720$, Region $_{2}: 163,714$, and Region $3: 156,470$. Again, CRM is utilized for comparison, in which the same discretization criterion is applied. As shown in Fig. 10, the H-H and V$\mathrm{V}$ polarized Bistatic RCS results of LCMT-DDM and CRM agree well with each other. The electric and magnetic current distribution of LCMT-DDM and CRM are further shown in Fig. 11 and Fig. 12. It can be seen that the current distribution also agrees well with each other.

The computational statistics of the two methods are shown in Table IV. FGMRES is used in LCMT-DDM and GMRES is used in CRM. In FGMRES, the convergence tolerance of the inner and outer iterations is set to be 0.01 and 0.001 , and in GMRES, the convergence tolerance is set to be 0.001 , which is the same as the outer iteration tolerance in FGMRES. It can be seen from table IV that, with the same discretization, LCMTDDM only needs one tenth of the iteration count required in

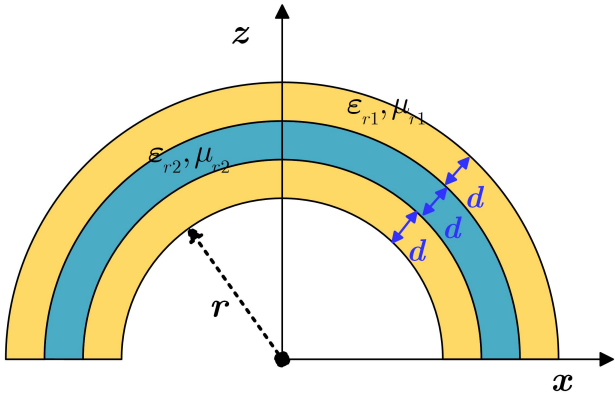

Fig. 9. The geometry and dimension of the three-layer dielectric radome.

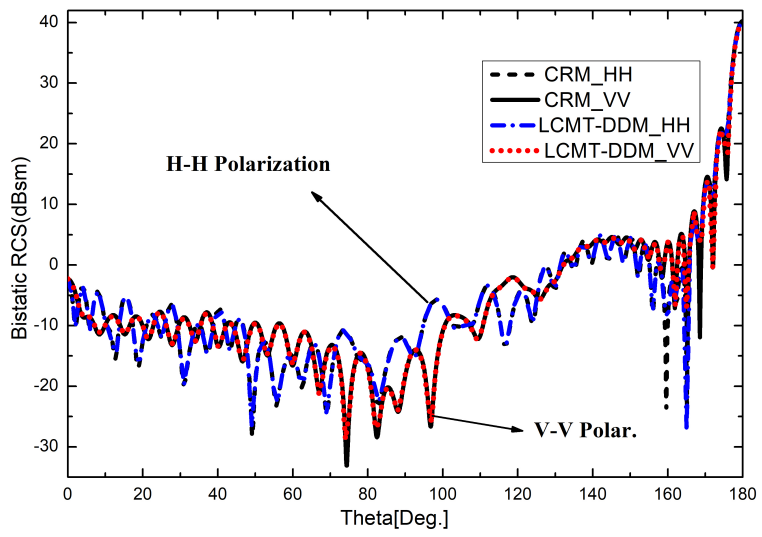

Fig. 10. The bistatic RCS of the three-layer dielectric radome.

CRM. Just like the previous example, the use of local coupling and the flexible preconditioner reduce the memory requirement and the CPU time of LCMT-DDM to the three fourth and the three fifth of those of CRM, respectively. It can be seen clearly that for large and complex models, LCMT-DDM has an obvious advantage over CRM.

TABLE IV

COMPUTATIONAL STATISTICS OF THE THREE-LAYER RADOME

\begin{tabular}{cccc}
\hline \hline & Time & Mem. & Iter. Num. \\
\hline LCMT-DDM: & $12,523 \mathrm{Sec}$. & $34.9 \mathrm{~GB}$ & 164 \\
\hline CRM: & $50,280 \mathrm{Sec}$. & $82.4 \mathrm{~GB}$ & 1755 \\
\hline \hline
\end{tabular}

\section{CONCLusions}

In this paper, a local coupling multi-trace domain decomposition method (LCMT-DDM) is presented to solve the EM scattering from multilayered dielectric objects. By a proper domain decomposition, the boundaries of all sub-domains can be made as touching-faces. Therefore, the interations between different sub-domains are all local coupling, leading to a sparse system matrix. In each sub-domain, the accurate EFIE and MFIE for dielectrics are applied as the governing equations. By enforcing the Robin transmission conditions (RTCs) on the boundaries, a diagonally dominant CFIE-like equation can be formulated for each sub-domain. According to this feature, the FGMRES method based on an inner-outer iteration is applied to solve the system matrix. Moreover, a preconditioner 

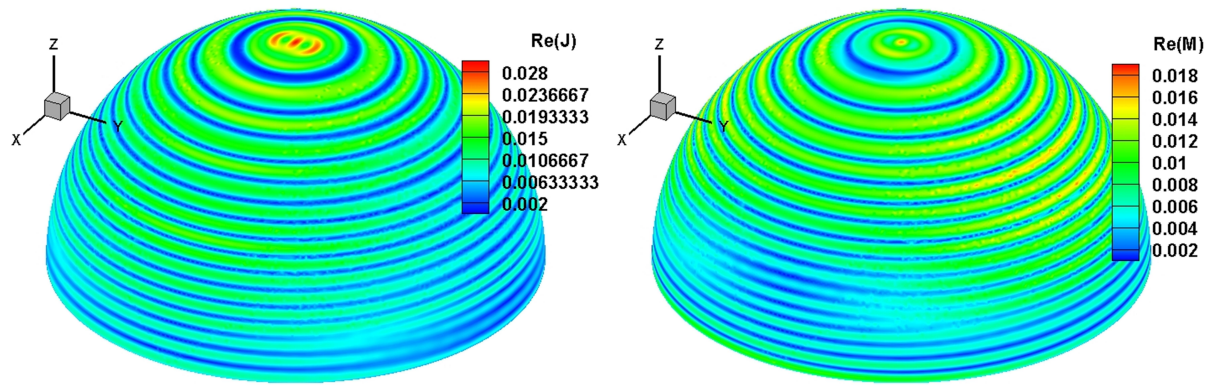

Fig. 11. The electric and magnetic currents of the three-layer dielectric radome from LCMT-DDM.

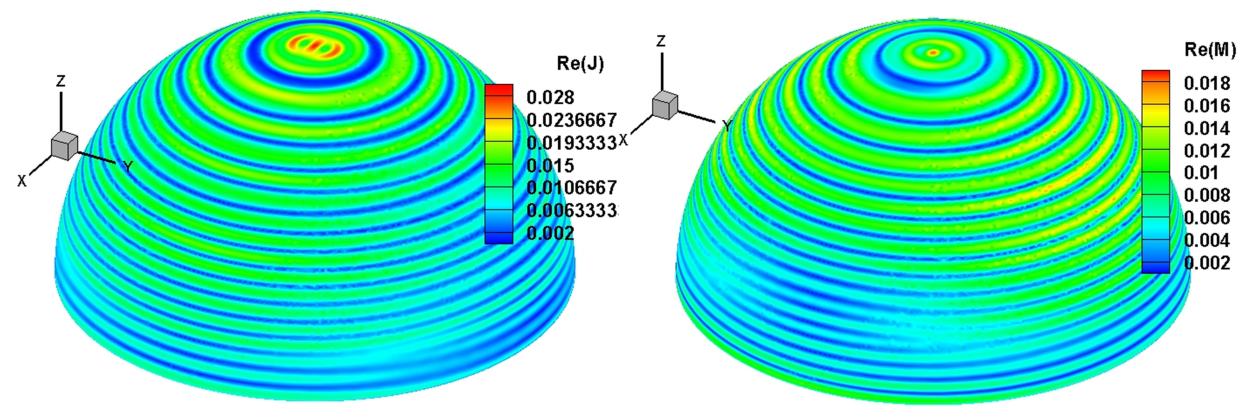

Fig. 12. The electric and magnetic currents of the three-layer dielectric radome from CRM.

that can be recursively constructed is proposed. Numerical results show that the proposed LCMT-DDM is superior over the existing CRM. With the same accuracy level, LCMTDDM has a better iterative convergence, less memory and CPU time consumption. The advantage is expected to be more significant when structures with more layers are considered. As future work, a symmetric formulation and the corresponding preconditioning technique will be explored to further increase the iterative convergence rate and reduce the computational requirements.

\section{REFERENCES}

[1] R. F. Harrington, Field Computation by Moment Methods. London, U. K.: Oxford Univ. Press, 1996.

[2] Y. Chang and R. Harrington, "A surface formulation for characteristic modes of material bodies," IEEE Trans. Antennas Propag., vol. AP-25, no. 6, pp. 789-795, Jun. 1977.

[3] Z. He, D. Z. Ding, and R. S. Chen, "An efficient marching-on-in-degree solver of surface integral equation for multilayer thin medium-coated conductors," IEEE Antennas Wireless Propag. Lett., vol. 15, pp. 14581461,2015

[4] M. S. Tong, "Meshfree solutions of volume integral equations for electromagnetic scattering by anisotropic objects," IEEE IEEE Trans. Antennas Propag., vol. 60, pp. 4249-4258, 2012.

[5] O. Saad and D. Jiao, "A new volume integral formulation for broadband 3 D circuit extraction in inhomogeneous materials with and without external electromagnetic fields," IEEE Trans. Microw. Theory Techn., vol. 61, pp. 4302-4312, 2013.

[6] Z. Q. Zhang and Q. H. Liu, "Three-dimensional weak-form conjugateand biconjugate-gradient FFT methods for volume integral equations," Microw. Opt. Tech. Lett., vol. 29, pp. 350-356, 2001.

[7] X. Q. Sheng, J. M. Jin, J. Song, W. C. Chew, and C. C. Lu, "Solution of combined-field integral equation using multilevel fast multipole algorithm for scattering by homogeneous bodies,"IEEE Trans. Antennas Propag., vol. 46, no. 11, pp. 1718-1726, 1998.

[8] Ö. Ergül and L. Gürel, "Novel electromagnetic surface integral equations for highly accurate computations of dielectric bodies with arbitrarily low contrasts," J. Comput. Phys., vol. 227, no. 23, pp. 9898-9912, 2008.
[9] P. Yla-Oijala, P. Taskinen, and J. Sarvas, "Surface integral equation method for general composite metallic and dielectric structures with junctions," Prog. Electromagn. Res., vol. 52, pp. 81-108, 2005.

[10] Y. Chu, W. C. Chew, J. Zhao, and S. Y. Chen, "A surface integral equation formulation for low-frequency scattering from a composite object," IEEE Trans. Antennas Propag., vol. 51, pp. 2837-2844, 2003.

[11] Z. Peng, X. C. Wang, and J. F. Lee, "Integral equation based domain decomposition method for solving electromagnetic wave scattering from non-penetrable objects," IEEE Trans. Antennas Propag., vol. 59, pp. 3328-3338, 2011.

[12] Z. Peng, K. H. Lim, and J. F. Lee, "Computations of electromagnetic wave scattering from penetrable composite targets using a surface integral equation method with multiple traces," IEEE Trans. Antennas Propag., vol. 61 , no. 1 , pp. 256-270, 2013.

[13] J. Hu, R. Zhao, M. Tian, H. P. Zhao, M. Jiang, X. Wei, and Z. P. Nie, "Domain Decomposition Method Based on Integral Equation for Solution of Scattering From Very Thin, Conducting Cavity," IEEE Trans. Antennas Propag., vol. 62, pp. 5344-5348, 2014.

[14] R. Zhao, J. Hu, H. P. Zhao, M. Jiang, and Z. P. Nie, "EFIE-PMCHWT based domain decomposition method for solving electromagnetic scattering from complex dielectric/metallic composite objects," IEEE Antennas Wireless Propag. Lett., vol. 16, pp. 1293-1296, 2017.

[15] P. Yla-Oijala and M. Taskinen, "Application of combined field integral equation for electromagnetic scattering by dielectric and composite objects," IEEE Trans. Antennas Propag., vol. 53, no. 3, pp. 1168-1173, 2005.

[16] Y. O. Pasi and T. Matti, "Improving conditioning of electromagnetic surface integral equations using normalized field quantities," IEEE Trans. Antennas Propag., vol. 55, no. 1, pp. 178-185, 2007.

[17] D. M. Solls, J. M. Taboada, O. Rubiños-López, and F. Obelleiro, "Improved combined tangential formulation for electromagnetic analysis of penetrable bodies," J. Opt. Soc. Amer. B, Opt. Phys., vol. 32, no. 9, pp. 1780-1787, Sep. 2015.

[18] Saad, Y. and Schultz, M. H, "GMRES: A generalized minimal residual algorithm for solving nonsymmetric linear systems," SIAM J. Sci. Comput., vol. 7, no. 3, pp. 856-869, 1986.

[19] Z. Peng, "A novel multitrace boundary integral equation formulation for electromagnetic cavity scattering problems," IEEE Trans. Antennas Propag., vol. 63, no. 10, pp. 4446-4457, 2015.

[20] R. Hiptmair and C. Jerezhanckes, "Multiple traces boundary integral formulation for Helmholtz transmission problems," Adv. Comput. Math., vol. 37, no. 1, pp. 39-91, Jul. 2012.

[21] K. Cools, F. P. Andriulli, and E. Michielssen "A Caldern multiplicative 
preconditioner for the PMCHWT integral equation," IEEE Trans. Antennas Propag., vol. 59, no. 12, pp. 34579-4587, Jul. 2011.

[22] L. Zhang, S. F. Tao, Z. H. Fan, and R. S. Chen, "Mixed innerouter iteration technique-based surface integral equations for fast solving EM scattering from penetrable objects," IEEE Trans. Antennas Propag., vol. 66, no. 9, pp. 4752-4758, 2018.

[23] Y. Saad, "A flexible inner-outer preconditioned GMRES algorithm," SIAM J. Sci. Comput., vol. 14, no. 2, pp. 461-469, 1993

[24] G. Alléon, M. Benzi, and L. Giraud, "Sparse approximate inverse preconditioning for dense linear systems arising in computational electromagnetics," Numer. Algorithms, vol. 16, pp. 1-15, 1997.

[25] J. Lee, J. Zhang, and C.-C. Lu, "Incomplete LU preconditioning for large scale dense complex linear systems from electromagnetic wave scattering problems," J. Comput. Phys., vol. 185, pp. 158-175, Feb. 2003.

[26] J. M. Song, C. C. Lu, and W. C. Chew, "Multilevel fast multipole algorithm for electromagnetic scattering by large complex objects," IEEE Trans. Antennas Propag., vol. 45, no. 10, pp. 1488-1493, 1997.

[27] S. M. Rao, D. R. Wilton, and A. W. Glisson, "Electromagnetic scattering by surfaces of arbitrary shape," IEEE Trans. Antennas Propag., vol. AP30, pp. 409-418, May 1982.

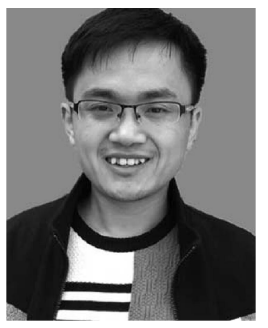

Ran Zhao (M'19) He received the B.S. degree from Anhui University (AHU), Hefei, China, in 2011, and the Ph.D. degree form University of Electronic Science and Technology of China (UESTC), Chengdu, China, in 2016. Since 2017, he has been an assistant professor with the School of electronic information and engineering, AHU. From 2019, he has been a Post-Doctoral Researcher with the Division of Computer, Electrical, and Mathematical Science and Engineering (CEMSE), King Abdullah University of Science and Technology (KAUST), Thuwal, Saudi Arabia. His research interests include numerical methods, especially full-wave methods in computational electromagnetics, electromagnetic scattering, and radiation.

He serves as a Reviewer for several journals, including the IEEE TRANSACTIONS ON ANTENNAS AND PROPAGATION, the IEEE TRANSACTIONS ON ELECTROMAGNETIC COMPATIBILITY, the IEEE ACCESS, and INTERNATIONAL JOURNAL OF NUMERICAL MODELLING: ELECTRONIC NETWORKS, DEVICES AND FIELDS.

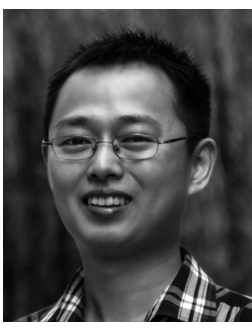

Yongpin Chen (S'06-M'12-SM'17) was born in Zhejiang, China, in 1981. He received the B.S. and M.S. degrees in microwave engineering from the University of Electronic Science and Technology of China (UESTC), Chengdu, China, in 2003 and 2006, respectively, and the Ph.D. degree in electrical and electronic engineering from The University of Hong Kong (HKU), Hong Kong, China, in 2012. From 2007 to 2008, he was a research assistant with the Electromagnetics and Optics Laboratory, HKU.

From 2015 to 2017, he was a Post-Doctoral Researcher with the Electro Science Laboratory, The Ohio State University, Columbus, OH, USA. Since 2012, he has been an Associate Professor with the School of Electronic Science and Engineering, UESTC. His current research interests include waves and fields in inhomogeneous media, computational electromagnetics, and antenna design.

Dr. Chen was a recipient of the General Assembly Young Scientists Award from the International Union of Radio Science in 2014, and also a recipient of the Best Young Scholar Paper Award from the Youth Academic Conference of the Chinese Institute of Electronics in 2017. He serves as a Reviewer for several journals, including the IEEE TRANSACTIONS ON ANTENNAS AND PROPAGATION, the IEEE TRANSACTIONS ON GEOSCIENCE AND REMOTE SENSING, the Journal of Computational Physics, and Physical Review A/E.

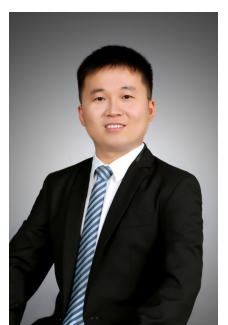

Xian-Ming Gu received the B.S. degree in mathematics and applied mathematics from Tangshan Teachers College, Tangshan, China, in 2011, and the Ph.D. degree in (computational) mathematics at the School of Mathematical Sciences, University of Electronic Science and Technology of China, Chengdu, Sichuan, China, in 2017. Since 2017, he has been an Assistant Professor with the School of Economic Mathematics, Southwestern University of Finance and Economics, Chengdu, Sichuan, China. He had ever joint in the Sandwich Ph.D. Position (8/2014-8/2016) of the Bernoulli Institute for Mathematics, Computer Science and Artificial Intelligence, University of Groningen, Groningen, the Netherlands. He is currently an Editor of for numerous publications, including Demonstratio Mathematica, Tbilisi Mathematical Journal and Journal of Mathematical Modeling. He has authored or coauthored over 44 research papers. His research interests include numerical linear algebra and applications to computational electromagnetics, parallel-in-time algorithms, and numerical solutions of (fractional) PDEs.

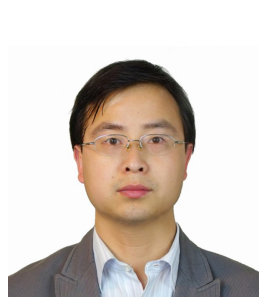

Zhixiang Huang (M16-SM18) was born in Anhui, China, in 1979. He received the B.S. and Ph.D. degree from Anhui University (AHU), China, in 2002 and 2007, respectively. Since 2008, he has been a Full Professor with the School of Electronic Information and Engineering, AHU.

From September 2010 to September 2011, he was a Visiting Scholar with Iowa State University, USA. From August 2013 to October 2013, he was a Visiting Professor with the University of Hong Kong. From February 2014 to February 2015, he was a Visiting Professor with the Beijing National Laboratory for Condensed Matter Physics, Institute of Physics, Chinese Academy of Sciences. He has co-authored one monograph on the symplectic finite-difference time-domain method and two book chapters for CRC Press and InTech Publishers. He has co-authored 70 peer-reviewed journal papers included in the Web of Science Core Collection. His current research interests include time-domain numerical methods, metamaterials, and active metamaterials.

Dr. Huang was the recipient of the Second Prize of Science and Technology from the Anhui Province Government, China, in 2015. He was also the recipient of the National Science Foundation for Outstanding Young Scholar of China in 2017 


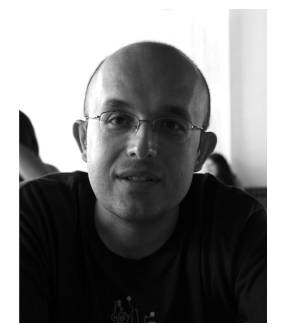

Hakan Bagci received the B.S. degree in Electrical and Electronics Engineering from the Bilkent University, Ankara, Turkey, in 2001; and the M.S. and Ph.D. degrees in Electrical and Computer Engineering from the University of Illinois at UrbanaChampaign (UIUC), Urbana, IL, USA, in 2003 and 2007, respectively. From June 1999 to July 2001, he worked as an Undergraduate Researcher with the Computational Electromagnetics Group, Bilkent University. From August 2001 to December 2006, he was a Research Assistant with the Center for Computational Electromagnetics and Electromagnetics Laboratory, UIUC. From January 2007 to August 2009, he was a Research Fellow with the Radiation Laboratory, University of Michigan, Ann Arbor, MI, USA. Since August 2009, he has been with the King Abdullah University of Science and Technology (KAUST), Thuwal, Saudi Arabia, where he is currently an Associate Professor of Electrical Engineering. His research interests include various aspects of theoretical and applied computational electromagnetics with emphasis on well-conditioned frequency and time domain integral equation formulations and their discretization, hybrid time domain integral and differential equation solvers, accurate, stable, and efficient marching schemes for time domain solvers, stochastic characterization of electromagnetic field and wave interactions on complex geometries, and solution of two and three dimensional electromagnetic inverse scattering problem using signal processing techniques. $\mathrm{He}$ authored or co-authored more than 90 journal papers and more than 200 contributions in conference proceedings. Dr. Bagci was the recipient of the 2008 International Union of Radio Scientists (URSI) Young Scientist Award and the 2004-2005 Interdisciplinary Graduate Fellowship from the Computational Science and Engineering Department, UIUC. His paper titled Fast and Rigorous Analysis of EMC/EMI Phenomena on Electrically Large and Complex Structures Loaded With Coaxial Cables was one of the three finalists (with honorable mention) for the 2008 Richard B. Schulz Best Transactions Paper Award given by the IEEE Electromagnetic Compatibility Society. He authored (as student) or co-authored (as student and advisor) 19 finalist/honorable mention papers in the student paper competitions at the 2005, 2008, 2010, 2014, 2015, 2016, 2017, 2018, and 2020 IEEE Antennas and Propagation Society International Symposiums and 2013, 2014, 2016, 2017, 2018, and 2019 Applied Computational Electromagnetics Society Conferences. Dr. Bagci is currently an Associate Editor for the IEEE Transactions on Antennas and Propagation, IEEE Journal on Multiscale and Multiphysics Computational Techniques, and IEEE Antennas and Propagation Magazine.

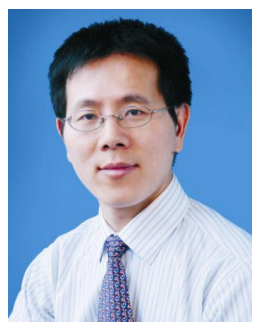

Jun Hu (M'06-SM'11) received the B.S., M.S., and $\mathrm{Ph} . \mathrm{D}$. degrees in electromagnetic field and $\mathrm{mi}-$ crowave technique from the University of Electronic Science and Technology of China (UESTC), Chengdu, in 1995, 1998, and 2000, respectively. During 2001, he was with the Center of Wireless Communication in the City University of Hong Kong, Kowloon, as a Research Assistant. During March to August in 2010, he was visiting scholar in the ElectroScience Laboratory of Department of ECE of the Ohio State University. He was Visiting Professor of City University of Hong Kong during Feb. to March in 2011. He is currently full Professor with the School of Electronic Science and Engineering of UESTC, IEEE senior member. Since September 2017, he has been Vice President of the UESTC. He also served as chairman of student activities committee in IEEE Chengdu Section during 2010-2016, chairman of IEEE Chengdu AP/EMC Joint Chapter during 2014-2016. He is the author or coauthor of over 300 technical papers, received 2004 best young scholar paper prize of Chinese Radio Propagation Society, and many best student papers awards. He was awarded as 2014 National Excellent Youth Fund by the NSFC, awarded as Chang Jing Scholar in 2016. He was the co-recipient of the 2018 IEEE AP-S Sergei A. Schelkunoff Transaction Paper Award.

His current research interests include integral equation methods in computational electromagnetics, electromagnetic scattering and radiation. 